\title{
The effects of CYP24A1 on clinicopathological features and the prognosis of pancreatic ductal adenocarcinoma
}

\author{
Wei Gao ${ }^{1,2 \#}$, Teng-Fei Wang ${ }^{1 \#}$, Wen Yi ${ }^{2}$, Wei-Min Tong ${ }^{3}$ \\ ${ }^{1}$ Department of Hepatobiliary Surgery, Aerospace Center Hospital, Aerospace Clinical Medical College of Beijing University, Beijing 100049, China; \\ ${ }^{2}$ Second Department of Hepatobiliary Surgery, Chinese PLA General Hospital, Chinese People's Liberation Army Medical School, Beijing 100853, \\ China; ${ }^{3}$ Department of Pathology, Institute of Basic Medical Sciences and Neuroscience Center, Chinese Academy of Medical Sciences and Peking \\ Union Medical College, Beijing 100005, China \\ Contributions: (I) Conception and design: W Gao; (II) Administrative support: W Gao; (III) Provision of study materials or patients: TF Wang, W Yi; \\ (IV) Collection and assembly of data: TF Wang ; (V) Data analysis and interpretation: W Gao, WM Tong; (VI) Manuscript writing: All authors; (VII) \\ Final approval of manuscript: All authors. \\ "These authors contributed equally to this work. \\ Correspondence to: Wei Gao. Department of Hepatobiliary Surgery, Aerospace Center Hospital, Aerospace Clinical Medical College of Beijing \\ University, 15 Yuquan Road, Haidian District, Beijing 100049, China. Email: drgaowei79@163.com.
}

Background: Pancreatic ductal adenocarcinoma (PDAC) is a type of exocrine pancreatic cancer that presents itself in the form of a highly malignant tumor. However, in the past few decades, with breakthroughs in the diagnosis and treatment of malignant tumors, there have yet to be satisfactory results for the diagnosis (early diagnosis) and treatment of PDAC. Therefore, the biological behavior of PDAC still requires more research to be understood. CYP24A1 is currently considered to be an essential part of vitamin D (VD) metabolism and increasingly reported to be associated with malignant tumors. Therefore, this study aims to explore the relationship between CYP24A1 and the clinicopathological features and prognosis of PDAC.

Methods: Seventy-three surgical PDAC cases were collected and follow-ups were made. The expression of CYP24A1 was obtained by the immunohistochemistry and the tissue FAXS cytometry (TFC) system. The related quantitative indices included the percentage of positive cells (\%) and the average staining intensity of the positive cells (in) in the cancer zone (C) and the adjacent non-cancer zone (ANC). The relationship between CYP24A1 and the clinicopathological parameters, as well as the prognosis of PDAC was then analyzed. Furthermore, Fluorescence quantitative PCR, Western-blot, siRNA, and phenotypic testing were implemented in the Pan-C1 PDAC cells.

Results: In normal pancreatic tissue, CYP24A1 was approximately "zero-expressed" in the exocrine glands. In the $\mathrm{C}$ and $\mathrm{ANC}$ zones, the expression of CYP24A1 increased significantly. In cases with a higher $\mathrm{C} \%$, the proportion of lymph node metastasis was lower $(\mathrm{P}=0.071)$; In cases with a higher $\mathrm{ANC} \%(\mathrm{P}=0.026)$ and higher ANCin ( $\mathrm{P}=0.079)$, the proportion of high differentiation was higher; in survival analysis, $\mathrm{C} \%$, Cin had a significant effect on survival and those with higher parameters had a lower risk of death. In the cell experiments, after CYP24A1 was silenced, the migration ability of PDAC cells did not change significantly and its proliferation $(\mathrm{P}=0.034)$ and invasion $(\mathrm{P}=0.002)$ ability decreased significantly.

Conclusions: CYP24A1 has a significant effect on the development and prognosis of PDAC.

Keywords: CYP24A1; pancreatic cancer; prognosis

Submitted Oct 12, 2019. Accepted for publication Jan 15, 2020.

doi: $10.21037 /$ tcr.2020.02.78

View this article at: http://dx.doi.org/10.21037/tcr.2020.02.78 


\section{Introduction}

Pancreatic ductal adenocarcinoma (PDAC) is a type of exocrine pancreatic cancer that presents itself in the form of a highly malignant tumor and its diagnosis (early diagnosis) and treatment have been highly concerning. In terms of imagelogical examination (1), gene level, and even the exosomes of cancer cells (2), people hope to discover PDAC lesions as early as possible; in terms of treatment, there have been many reports on pancreatic cancer surgery and various comprehensive treatments. However, in the past few decades, in the case of breakthroughs in the diagnosis and treatment of malignant tumors, there have been no satisfactory results in the diagnosis (early diagnosis) and treatment of PDAC. Therefore, the biological behavior of PDAC still requires more research to understand the disease.

CYP24A1 is currently considered to be an essential part of VD metabolism. As an exogenous substance, VD is a fat-soluble vitamin and needs to undergo a series of activations in the human body to form active VD-calcitriol $(1,25(\mathrm{OH}) 2 \mathrm{D} 3)$, while CYP24A1 inactivates $1,25(\mathrm{OH}) 2 \mathrm{D} 3$ by hydroxylation. 1,25(OH)2D3 binds to the VDR (VD receptor) and can express its regulatory functions in gene expressions. 1,25(OH)2D3 can regulate the level of calcium and phosphorus in the body and maintain the normal bone function. Its role in the tumor has received growing attention in recent years. At present, 1,25(OH)2D 3 is considered to have an antitumor effect. As an essential part of the regulation of VD activity, CYP24A1 is increasingly reported to be associated with malignant tumors, such as breast cancer (3), esophageal cancer (4), colorectal cancer (5), prostate cancer (6), ovarian cancer (7), and its role in tumorigenesis. Close attention has also increasingly been paid to the relevant progression and prognosis. In this study, we analyzed the relationship between the expression of CYP24A1 and the clinicopathological characteristics of PDAC, and proceeded with the survival analysis of PDAC patients. In PDAC cell experiments, the CYP24A1 effects were understood through RNA interference. Thereafter, the roles of CYP24A1 in the occurrence, progression, and prognosis of PDAC were discussed.

\section{Methods}

\section{Patients and samples}

Seventy-three PDAC specimens from surgery were collected; two experienced pathologists confirmed all the cases as PDAC. All subjects received surgery for the first time; no chemotherapy or radiotherapy was conducted prior to surgery. The HE staining section of the paraffin block selected Cancer tissue, adjacent non-cancer (ANC) tissue, and normal pancreas tissue. Clinicopathological parameters were collected, including age, gender, tumor size, tumor location, tumor differentiation, clinical-pathological stage (American Joint Commission on Cancer 8th edition), venous and/or perineural invasion. Among the 73 patients, there were 47 males and 26 females and the age range was from 36 to 82 years old (median: 58 years old), 42 of which $<60$ years old and 31 of which $>60$ years old. Among the clinicopathological parameters, the differentiation status was high at 31 cases, poor differentiation at 42 cases; tumor size (maximum diameter $\leq 2 \mathrm{~cm}$ and $>2 \mathrm{~cm}$ group): the smaller one was 11 cases and the larger one was 62 cases; tumor location: 48 in the head of the pancreas, 25 in the body and tail; lymph node metastasis: 43 were negative and 23 were positive, 7 of which did not obtain peripancreatic lymph node specimens; clinical-pathological staging, according to the eighth edition of the AJCC PDAC staging criteria, 62 cases in stage I and II, 11 cases in stage III and $\mathrm{IV}$, and neurological and vascular invasion was negative in 37 cases and positive in 36 cases. The patient's survival was reviewed by a telephone follow-up. The final follow-up date was June 20, 2018, in which 60 died, 9 survived, and 4 could not be followed up with.

All cases were obtained from the second department of Hepatobiliary Surgery at the General Hospital of PLA, CHINA. The Ethical Committee of the General Hospital of PLA approved the present study (ID: S2016-098-02) and all subjects signed informed consent prior to the study.

\section{Immunobistochemistry and the quantitative analysis of immunobistochemical staining}

Operating conventional immunohistochemical staining, after a preliminary test, the determined dilution concentration of the primary antibody (21582-1-AP, Proteintech Group Inc, WUHAN, CHINA) was 1:50. The PBS solution was used instead of the primary antibody as a negative control and the kidney tissue paraffin section was used as a positive control.

The TFC system was used for establishing the individualized analysis files. Then, through the highdefinition scanning (TissueFAXS4.2.6245.1031) and analysis process (StrataQuest ALL 5.0.1.317), the quantitative indexes of CYP24A1 expression (percentage of positive cells-\%, mean intensity-in) in the C and ANC zones of 
PDAC were obtained $(8,9)$. In this experiment, we ensured that the number of cells that were recruited in every area was $\geq 5,000$.

\section{CYP24A1 silenced and the phenotypic analysis of PDAC PanC-1 cells}

The Institute of Basic Medical Sciences, Chinese Academy of Medical Sciences, and Peking Union Medical College donated the PDAC PanC-1 cell. The CYP24A1-siRNA design was completed by GenePharma, Inc. Suzhou, CHINA. There were three preselected interference sequences:

\section{* si608: GCUGCAGAUUCUCUGGAAATtUUUC CAGAGAAUCUGCAGCTT; \\ * si1045: GCCUCGUGUUGUAUGAgAatTUUC UCAUACAACACGAGGCTT; \\ * si1599: gCAaCAguUCugggugaauttauU CACCCAGAACUGUUGCTT.}

The silencing effect was studied by fluorescence quantitative PCR and Western Blot experiments and the interference sequence was determined as si1599. The ability changes of proliferation, migration, and invasion of PanC-1 cells before and after being stopped was analyzed by CCK8 (cell counting kit-8), Transwell migration, and Transwell invasion experiments.

\section{PanC-1 cell transfection}

The complete medium was changed to the serum-free medium half an hour before the transfection. Solution A: SiRNA 100 pmol was added into the serum-free medium of 50 UL Opti-MEM (31985-070, Invitrogen Life Technologies (GIBCO $\left.{ }^{\mathrm{TM}}\right)$ ), gently mixed, and placed at room temperature for 5 minutes. Solution B: Lipofectamine 2000 [780373, Invitrogen Life Technologies (Lipofectamine $\left.^{\mathrm{TM}}\right)$ ] was gently mixed before use. Liposomes $5 \mu \mathrm{L}$ was added to $50 \mu \mathrm{L}$ of the Opti-MEM serum-free medium, gently mixed, and placed at room temperature for 5 minutes. Solution B was added to solution A, mixed gently, and placed steadily at room temperature for 20 minutes. The mixture was evenly dispersed and slowly dripped into the cells in the 6-well plate. Thereafter, it was incubated at $37^{\circ} \mathrm{C}$ for $4-6$ hours. The serum-free medium was then replaced with the complete medium and left to culture. After 36-48 hours of transfection, the mRNA level detection was performed and the protein level detection was performed after 48-72 hours of transfection.

\section{Fluorescence quantitative PCR test}

The total RNA was isolated from the cultured cells by RNA-Trizol (G3013, Servicebio, Wuhan, China). The RNA was then reverse transcribed and subjected to the SYBR Green-based RT-PCR analysis. The digested cell suspension was centrifuged at $10,000 \mathrm{r} / \mathrm{min}$ and $0.2 \mathrm{~mL}$ of chloroform was added to every milliliter of the reagent lysis sample for pyrolysis operation. RNA was obtained by washing with an enzyme-free RNA washing solution on ice at $4{ }^{\circ} \mathrm{C}$ and centrifugation at $10,000 \mathrm{r} / \mathrm{min}$ for 5 minutes. Before adding reverse transcriptase, the mixture was dried at $70{ }^{\circ} \mathrm{C}$ for 3 minutes. After removing immediately, the ice water bath was transferred to allow the temperature inside and outside the tube to become uniform. $0.5 \mu \mathrm{L}$ of reverse transcriptase was added and left for 60 minutes in the water bath at $37^{\circ} \mathrm{C}$ and was left to dissolve completely for 5 minutes at room temperature to reverse-transcribe it into cDNA. Actin B and SYBR Green 1 dye were used as a template; primers and reverse transcription products were added to the reaction system to make the total volume up to $20 \mu \mathrm{L}$. The reaction conditions were as follows: predenaturation at $93{ }^{\circ} \mathrm{C}$ for 2 minutes, denaturation at $93{ }^{\circ} \mathrm{C}$ for 1 minute, and annealing at $55^{\circ} \mathrm{C}$ for 2 minutes with a total of 40 cycles. The reverse transcriptase kit K1622 was purchased from Thermo and the SYBR Green Master (Rox Reference Dye) was purchased from Roche. The results were processed by the $\Delta \Delta \mathrm{CT}$ method.

\section{Western blot experiment}

Adherent cells were centrifuged for 10 minutes at 12,000 $\mathrm{r} / \mathrm{min}\left({ }^{\circ} \mathrm{C}\right)$ and supernatants were collected. The BCA protein concentration measuring kit was used to measure protein concentration. SDS-PAGE electrophoresis was used until the bromophenol blue had run out and then we proceeded to terminate the electrophoresis and transfer the membrane with $300 \mathrm{~mA}$ constant current for half an hour. The transferred membrane was blocked with $5 \%$ skim milk for 1 hour at room temperature on a decolorization shaking table, placed in a diluted $(1: 1,000)$ primary antibody (AntiCYP24A1, ab137116, ABCAM), and incubated overnight at $4{ }^{\circ} \mathrm{C}$. It was then washed three times with TBST on a decolorization shaking table at room temperature for 5 minutes each time. The secondary antibody was diluted 3,000 times with TBST, incubated for 30 minutes at room temperature, and washed three times with TBST on a decolorization shaking table at room temperature for 
5 minutes each time. After exposure, the film was scanned and archived. Photoshop was used to decolorize and Alpha software processing system was used to analyze the optical density of the target band.

\section{CCK-8 cell proliferation experiment}

The logarithmic growth phase cells were trypsinized and prepared into cell suspensions, seeded in 96-well plates at $3,000-5,000$ cells per well with $100 \mu \mathrm{L}$ per well, and placed in a CO2 $(5 \%)$ incubator at $37{ }^{\circ} \mathrm{C}$ overnight. The marginal holes were filled with sterile PBS. The control group and the transfection group each had 6 wells, $10 \mu \mathrm{L}$ of CCK-8 solution was added in all wells, we then gently tapped the plate and mixed for 2 hours in the incubator. The $450 \mathrm{~nm}$ light absorption was measured by enzyme labeling. The CCK-8 kit developed by Dojindo Laboratories was used in the experiment.

\section{Transwell migration and invasion experiment}

The transwell plate chamber was $200 \mu \mathrm{L}$ of the serum-free medium. $500 \mu \mathrm{L}$ of the complete medium containing $20 \%$ FBS was added to the lower chamber and the cell suspension was added to the upper chamber (according to trial test, $0.2 \times 10^{5}$ cells were added to the migration experiment and $1 \times 10^{5}$ cells were added to the invasion test). Transwell plates were cultured in a $\mathrm{CO} 2$ (content $5 \%$ ) incubator at $37{ }^{\circ} \mathrm{C}$ for 24 hours (invasive experiment for 48 hours). Then the chamber was taken out, the culture medium was washed with PBS, and stained with crystal violet for 10 minutes. The surface of the chamber was washed with tap water. The cells on the inoculated side of the upper chamber were erased with a cotton swab and the number of cells passing through the chamber was photographed and analyzed under a 100-fold microscope. Preparation of transwell invasion wells: Dilute BD matrigel and the serum-free medium at a ratio of $1: 8$, pipet $200 \mu \mathrm{L}$ into the upper chamber of the transwell and place in the incubator for at least 2 hours.

\section{Statistical analysis}

SPSS20 (Statistical Package for the Social Science) computer statistical analysis software was used to process the experimental data. The quantitative parameters of the obtained CYP24A1 expression were visually discretized under the SPSS "Transform"-"Visual Banning" menu and the data obtained were divided into low and high groups at a split point based on "equal percentiles based on scanned cases". The Chi-square test was performed with the staining parameters and the clinicopathological parameters of PDAC cases. Combined with the follow-up situation, the clinicopathological parameters and the parameters obtained from the staining analysis were analyzed by a Log-Rank test and Cox regression single factor analysis to understand the relationship between each parameter and prognosis and then the parameters related to prognosis were considered in the screened staining analysis. Furthermore, the factors related to prognosis were analyzed by Cox regression multifactor analysis. $\mathrm{P}<0.05$ indicates that the difference was statistically significant.

\section{Results}

\section{Relevant results of immunobistochemical parameters}

\section{Preliminary interpretation of immunohistochemical staining}

In normal pancreatic tissue, CYP24A1 has almost no positive expression in the pancreatic exocrine glandcatheter epithelium and glandular epithelial tissue; however, in normal pancreatic tissue, CYP24A1 is clearly expressed in the endocrine-islet tissue of the pancreas. In the $\mathrm{C}$ and ANC zones, CYP24A1 was expressed in different degrees in the pancreatic exocrine gland cells (Figure 1). This result is consistent with previous reports (10).

The expression of CYP24A1 in pancreatic exocrine gland cells was significantly changed in the adjacent noncancerous tissue compared with normal pancreatic tissue. With the occurrence of ductal metaplasia and the chaotic arrangement of cells in the pancreatic exocrine gland epithelium, the expression of CYP24A1 in exocrine cells was more positive than that in normal pancreatic regions. Tumors proliferate, invade surrounding tissue, and metastasize to a distance. Thus, their "sphere of influence" increases; this process must be through its adjacent tissue. The expression of the gene in the ANC zone should have a certain significance for understanding its role in tumorigenesis and progression.

\section{The relationship between CYP24A1 and clinicopathological parameters of PDAC}

Preliminary conditions showed that the CYP24A1 approximate was "zero expressed" in normal pancreatic exocrine tissue and was positive in both $\mathrm{C}$ and $\mathrm{ANC}$ zones, suggesting that CYP24A1 may have some significance in the occurrence and progression of PDAC. The clinicalpathological parameters of PDAC cases were analyzed and 

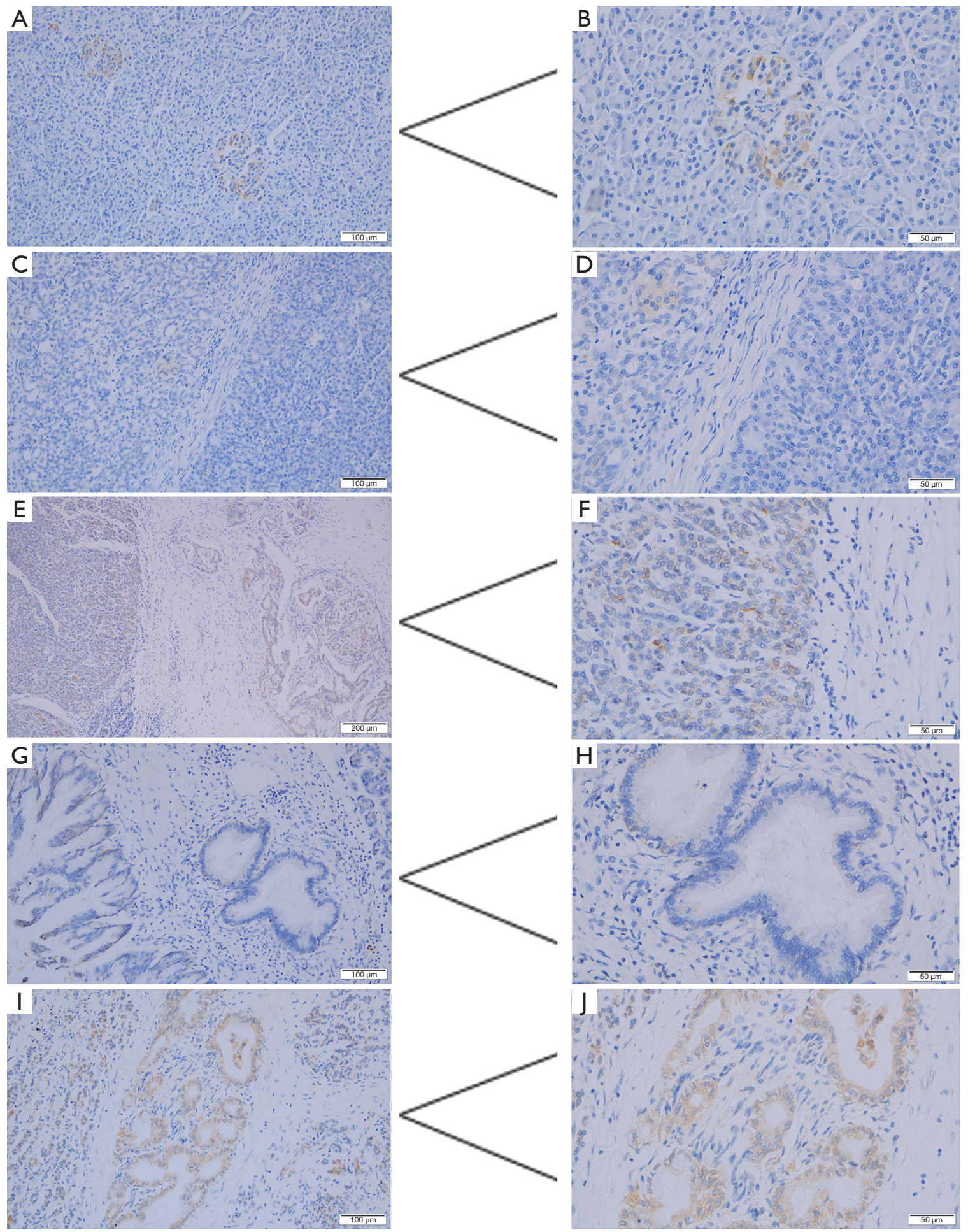

Figure 1 CYP24A1 expression in PDAC tissue, adjacent non-cancer tissue, and normal pancreas tissue. (A,B) In normal pancreatic tissue, CYP24A1 was zero expressed in exocrine glands and significantly expressed in islets. (C,D) Low expression of CYP24A1 in ANC zone of PDAC. (E,F) High expression of CYP24A1 in ANC zone of PDAC. (G,H) Low expression of CYP24A1 in the C zone of PDAC. (I,J) High expression of CYP24A1 in the C zone of PDAC. Original magnification $\times 100$ (E), $\times 200$ (A, C, G, and I) and ×400 (B, D, F, H, and J). PDAC, pancreatic ductal adenocarcinoma. 
listed in Table 1. The results showed that $\mathrm{C} \%$ was associated with lymph node metastasis and the proportion of metastasis was lower in cases with higher $\mathrm{C} \%(\mathrm{P}=0.071)$. ANC\% was associated with $\mathrm{PDAC}$ differentiation and the proportion of higher differentiation was higher in cases with higher ANC\% $(\mathrm{P}=0.026)$; ANCin was associated with PDAC differentiation and the proportion of higher differentiation was higher in cases with higher ANCin ( $\mathrm{P}=0.079)$.

\section{Survival analysis}

In the univariate analysis of the survival data, tumor differentiation, size, location, lymph node metastasis, and neurovascular invasion of PDAC in clinicopathological indices (Table 2) were not statistically significant but the trend of their impact on survival was consistent with the opinions that we currently consider. Unexpectedly, in the clinicopathological stages, the survival time of patients with an earlier stage (stage I and II) is shorter than that of patients with a later stage (stage III and IV). It may be that the sample size of patients at a later stage is too small, but to some extent, it also suggests that for resectable PDAC patients, although the stage is more progressed, surgical resection is of considerable significance for improving survival. Among the related indexes of CYP24A1 expression (Table 3), there was no statistical significance in the univariate survival analysis.

\section{Cox regression multivariate analysis}

Through the univariate analysis of the survival parameter, the influence of $\mathrm{C} \%$ and $\mathrm{Cin}$ on the survival time could be included in the multivariate analysis. A Cox regression multivariate analysis was performed on the staining and clinicopathological parameters. Pathological parameters included age, differentiation level, tumor size, location, lymph node metastasis, clinical-pathological stage, and neurovascular status. In the cox multivariate analysis of the survival data, it was further confirmed that $\mathrm{C} \%(\mathrm{P}=0.023)$ and $\mathrm{Cin}(\mathrm{P}=0.026)$ had statistically significant positive associations with survival (Figure 2). Those with higher parameters had a lower risk of death, with HR (95\% CI) of $0.530(0.307-0.917)$ and $0.535(0.309-0.927)$.

\section{PDAC cell test}

\section{Confirmation of CYP24A1-siRNA interference sequence in PanC-1 cells}

PanC-1 cells were transfected with three pre-selected sequences and the silencing effect of each interference sequence on the CYP24A1 gene was determined by fluorescence quantitative PCR (Primer sequence in Table 4) and Western Blot (Figure 3). Thereby si1599 was determined as the interference sequence.

\section{Phenotypic changes of PanC-1 cells after CYP24A1 silenced \\ Changes in proliferation ability of PanC-1 cells after CYP24A1 silenced}

After the completion of the CCK-8 experiment, the OD values of each well of the experimental group (interference group) and the negative control group were obtained: siRNA group was $0.4058, \mathrm{NC}$ was $0.4285 ; \mathrm{P}=0.06$.

\section{Changes of the migration ability of PanC-1 cells after CYP24A1 was stopped}

After the cell migration transwell test, the number of porous migration cells in the experimental group and the negative control group was obtained (Figure 3C), that is $228.0 \pm 18.4$ in the NC group and $228.2 \pm 19.9$ in the siRNA group $(\mathrm{P}=0.987)$. The result suggests that the migration ability of PanC-1 cells had no significant change after CYP24A1 was silenced.

\section{Changes of the invasion ability of PanC-1 Cells after CYP24A1 was silenced}

After the transwell invasion test, the number of porous infiltrating cells was obtained, that is $1,136.8 \pm 51.4$ in the $\mathrm{NC}$ group and $843.0 \pm 66.9$ in the siRNA group, $\mathrm{P}=0.002$. The result suggested that the invasion ability of PanC-1 cells decreased after CYP24A1 was silenced.

\section{Discussion}

In recent years, vitamins, as a necessary substance for the human body, have attracted much attention for their role in malignant tumors (11). As the only vitamin that can be synthesized by the human body in a small amount, VD is considered to be a steroid and its effect is more concerning than other vitamins. At present, 1,25(OH)2D3 is considered to have an anti-tumor effect, which can affect the biological behavior of tumors in many aspects such as proliferation, differentiation, apoptosis, angiogenesis, and mesenchymal transformation (12-14). However, there are still many uncertain conclusions about the role of VD in tumors (15). It has been reported that $\mathrm{VD}$ and its related factors may be involved in the occurrence, progression, and prognosis of PDAC patients $(16,17)$ but these views are still inconsistent. CYP24A1 is the key to the regulation of VD biological activity and the reports about its functions in tumors are increasing. However, there are few reports on the 
Table 1 Relationship between CYP24A1 and clinical pathological parameters of PDAC

\begin{tabular}{|c|c|c|c|}
\hline Clinicopathological indexes & Number & $\chi^{2}$ & $\mathrm{P}$ \\
\hline \multicolumn{4}{|l|}{$\mathrm{C} \%$} \\
\hline Differentiation & & 0.658 & 0.417 \\
\hline High & $14 / 17$ & & \\
\hline Poor & 23/19 & & \\
\hline Tumor size & & 0.077 & 0.781 \\
\hline$\leq 2 \mathrm{~cm}$ & $6 / 5$ & & \\
\hline$>2 \mathrm{~cm}$ & $31 / 31$ & & \\
\hline Tumor location & & 0.026 & 0.871 \\
\hline Head & $24 / 24$ & & \\
\hline Body and tail & $13 / 12$ & & \\
\hline Lymph node metastasis $^{a}$ & & 3.270 & $0.071^{\mathrm{b}}$ \\
\hline No & $18 / 25$ & & \\
\hline Yes & $15 / 8$ & & \\
\hline Staging (AJCC stage) & & 1.467 & 0.226 \\
\hline I, II & $29 / 32$ & & \\
\hline III, IV & $8 / 4$ & & \\
\hline Neurovascular invasion & & 0.013 & 0.908 \\
\hline No & $19 / 18$ & & \\
\hline Yes & $18 / 18$ & & \\
\hline \multicolumn{4}{|l|}{ Cin } \\
\hline Differentiation & & 0.658 & 0.417 \\
\hline High & $14 / 17$ & & \\
\hline Poor & $23 / 19$ & & \\
\hline Tumor size & & 0.869 & 0.351 \\
\hline$\leq 2 \mathrm{~cm}$ & $7 / 4$ & & \\
\hline$>2 \mathrm{~cm}$ & $30 / 32$ & & \\
\hline Tumor location & & 0.430 & 0.512 \\
\hline Head & $23 / 25$ & & \\
\hline Body and tail & $14 / 11$ & & \\
\hline Lymph node metastasis & & 0.601 & 0.438 \\
\hline No & $20 / 23$ & & \\
\hline Yes & $13 / 10$ & & \\
\hline $\begin{array}{l}\text { Clinical pathological staging } \\
\text { (A stage) }\end{array}$ & & 1.467 & 0.226 \\
\hline I, II & $29 / 32$ & & \\
\hline III, IV & $8 / 4$ & & \\
\hline
\end{tabular}

Table 1 (continued)
Table 1 (continued)

\begin{tabular}{|c|c|c|c|}
\hline Clinicopathological indexes & Number & $\chi^{2}$ & $\mathrm{P}$ \\
\hline Neurovascular invasion & & 0.124 & 0.724 \\
\hline No & $18 / 19$ & & \\
\hline Yes & $19 / 17$ & & \\
\hline \multicolumn{4}{|l|}{ ANC\% } \\
\hline Differentiation & & 4.981 & $0.026^{c}$ \\
\hline High & $11 / 20$ & & \\
\hline Poor & $26 / 16$ & & \\
\hline Tumor size & & 0.869 & 0.351 \\
\hline$\leq 2 \mathrm{~cm}$ & $7 / 4$ & & \\
\hline$>2 \mathrm{~cm}$ & $30 / 32$ & & \\
\hline Tumor location & & 0.026 & 0.871 \\
\hline Head & $24 / 24$ & & \\
\hline Body and tail & $13 / 12$ & & \\
\hline Lymph node metastasis & & 0.871 & 0.351 \\
\hline No & $21 / 22$ & & \\
\hline Yes & $14 / 9$ & & \\
\hline $\begin{array}{l}\text { Clinical pathological staging } \\
\text { (A stage) }\end{array}$ & & 0.467 & 0.494 \\
\hline I, II & $32 / 29$ & & \\
\hline III, IV & $5 / 7$ & & \\
\hline Neurovascular invasion & & 0.124 & 0.724 \\
\hline No & $18 / 19$ & & \\
\hline Yes & $19 / 17$ & & \\
\hline \multicolumn{4}{|l|}{ ANCin } \\
\hline Differentiation & & 3.091 & $0.079^{b}$ \\
\hline High & $12 / 19$ & & \\
\hline Poor & $25 / 17$ & & \\
\hline Tumor size & & 1.063 & 0.303 \\
\hline$\leq 2 \mathrm{~cm}$ & $4 / 7$ & & \\
\hline$>2 \mathrm{~cm}$ & $33 / 29$ & & \\
\hline Tumor location & & 0.430 & 0.512 \\
\hline Head & $23 / 25$ & & \\
\hline Body and tail & $14 / 11$ & & \\
\hline Lymph node metastasis & & 0.067 & 0.796 \\
\hline No & $22 / 21$ & & \\
\hline Yes & $11 / 12$ & & \\
\hline
\end{tabular}

Table 1 (continued) 
Table 1 (continued)

\begin{tabular}{lccc}
\hline Clinicopathological indexes & Number & $\chi^{2}$ & $\mathrm{P}$ \\
\hline $\begin{array}{l}\text { Clinical pathological staging } \\
\text { (A stage) }\end{array}$ & & 0.467 & 0.494 \\
I, II & $32 / 29$ & & \\
III, IV & $5 / 7$ & & \\
Neurovascular invasion & & 0.013 & 0.908 \\
No & $19 / 18$ & & \\
Yes & $18 / 18$ & & \\
\hline
\end{tabular}

${ }^{a}$, among 73 cases, 7 cases had no lymph node in pathological report; ${ }^{b}$, difference is almost statistically significant; ${ }^{c}$, difference is statistically significant. PDAC, pancreatic ductal adenocarcinoma.

relationship between CYP24A1 and PDAC in real-world cases, especially the relationship between the expression of CYP24A1 and the prognosis of patients with PDAC, which has not yet been reported. Through this study, CYP24A1 functions in PDAC were investigated further.

In the study results, cases with a higher $\mathrm{C} \%$ had a low proportion of lymph node metastasis $(\mathrm{P}=0.071$ : nearly statistically significant). If the metabolic pathway of VD is still used to explain this situation and the high expression of CYP24A1 leads to VD inactivation, then the tumorinhibition of VD is weakened and the proportion of lymph node metastasis should be increased, which is contrary to the experimental results.

It has been reported that the proportion of lymph node metastasis in colorectal cancer patients with a high expression of CYP24A1 is high (5), which is inconsistent with the results of this present experiment. In another study, although the relationship between VDR expression and lymph node metastasis of PDAC was not statistically significant (18), the proportion of lymph node metastasis in patients with a high expression of VDR tended to be lower. Therefore, the relationship between CYP24A1 and VD metabolic pathway in the lymph node metastasis of PDAC remains unclear, and depends on further research.

The results suggested that the expression of CYP24A1 in the ANC zone was related to the differentiation of PDAC. In both $\mathrm{T} \%(\mathrm{P}=0.026)$ and Tin $(\mathrm{P}=0.079)$, among cases with high staining parameters, the proportion of highly differentiated cancer is large, which suggested that the expression of CYP24A1 is more helpful to the high differentiation of pancreatic exocrine gland cells in the process of malignant transformation. In terms of the relationship between VD and tumor differentiation, relevant reports have shown that VD can induce differentiation $(19,20)$. If the effect of CYP24A1 on PDAC differentiation is considered through the VD metabolic pathway, the overexpression of CYP24A1 will promote the inactivation of $1,25(\mathrm{OH}) 2 \mathrm{D} 3$, thus, the induction and differentiation function of VD for cancer cells is reduced. The result should be a high proportion of low differentiation. This contradictory result shows that if CYP24A1 plays a role in cell differentiation through other pathways (apart from VD), it then deserves further study.

In the survival data, $\mathrm{C} \%$ and $\mathrm{Cin}$ are protective factors for postoperative survival, suggesting that the relatively high expression of CYP24A1 in cancer cells may be a protective factor, which is different from CYP24A1 in other tumor survival assessments $(5,19)$ and if the VD pathway is passed, it is not reasonable to explain the protective effect of CYP24A1 on postoperative survival.

In the cell test, the proliferation and invasion ability of PDAC cells after CYP24A1 was silenced decreased significantly, indicating that CYP24A1 has a certain degree of influence on the biological behavior of PDAC cells and that the test results suggest that in the absence of $\mathrm{VD}$, the changes of CYP24A1 expression also have an impact on the phenotype of PDAC cells. In the studies showing the effects of CYP24A1 on tumor cells, breast cancer cells (21) demonstrated that the knocking down of CYP24A1 impacted the metabolism of $1,25(\mathrm{OH}) 2 \mathrm{D} 3$ and the geneexpression profile was altered in some cancer-associated genes. Furthermore, CYP24A1-induced vitamin D (VD) insufficiency promotes breast cancer growth. Höbaus et al. reported their studies on human colorectal cancer cellHT29 xenograft, investigated by feeding mice VD and soy (a kind of CYP24A1 inhibitor). They assumed that a diet with VD and/or soy could inhibit tumor growth only in conditions of CYP24A1 common expressions. Nevertheless, in conditions of CYP24A1 overexpression, the tumor volume of mice with a high VD diet was bigger than in a low VD diet. During CYP24A1 overexpression, the inhibition of the CYP24A1-inhibitor to the tumor proliferation had not been reflected (22). Therefore, CYP24A1 affects the biological behavior of tumor cells, however, the exact situation remains unclear.

There were some limitations to this study. Firstly, there were insufficient data about the multivariable analysis of the relationship between CYP24A1 and clinicopathological 
Table 2 Univariate analysis of clinicopathological parameters with overall survival

\begin{tabular}{|c|c|c|c|c|}
\hline Clinicopathological parameters & \multicolumn{2}{|l|}{ Log-Rank test } & \multicolumn{2}{|c|}{ Cox analysis } \\
\hline \multicolumn{5}{|l|}{ Age } \\
\hline$\leq 60$ years & $19.0(15.3-22.7)$ & 0.083 & $1.55(0.93-2.58)$ & 0.096 \\
\hline$>60$ years & $13.0(11.5-14.5)$ & & & \\
\hline Female & $14.0(9.9-18.1)$ & & & \\
\hline \multicolumn{5}{|l|}{ Cancer location } \\
\hline Head & $13.0(9.5-16.6)$ & 0.828 & $0.94(0.55-1.63)$ & 0.833 \\
\hline Poor & $13.0(11.7-14.3)$ & & & \\
\hline \multicolumn{5}{|l|}{ Size } \\
\hline$\leq 2 \mathrm{~cm}$ & $20.0(14.2-25.6)$ & 0.350 & $1.44(0.65-3.17)$ & 0.368 \\
\hline$>2 \mathrm{~cm}$ & $14.0(11.1-16.9)$ & & & \\
\hline \multicolumn{5}{|l|}{ Lymph node } \\
\hline Negative & $16.0(11.4-20.1)$ & 0.411 & $1.26(0.72-2.20)$ & 0.428 \\
\hline Positive & $12.0(9.7-14.3)$ & & & \\
\hline \multicolumn{5}{|l|}{ Staging } \\
\hline
\end{tabular}

Table 3 Univariate analysis of staining parameters with overall survival

\begin{tabular}{lccc}
\hline \multirow{2}{*}{ CYP24A1 expression } & \multicolumn{1}{c}{ Log-Rank test } & & \multicolumn{2}{c}{ Cox analysis } \\
\cline { 2 - 4 } C\% & Median survival time $(95 \% \mathrm{Cl})$ & $\mathrm{P}$ & $\mathrm{HR}(95 \% \mathrm{Cl})$ \\
\cline { 2 - 4 } Cin & $13.0(10.1-15.9)$ & 0.090 & $0.65(0.39-1.09)$ \\
& $17.0(11.3-22.7)$ & & $0.66(0.40-1.10)$ \\
ANC\% & $13.0(10.6-15.4)$ & 0.098 & $0.97(0.58-1.60)$ \\
ANCin & $17.0(11.2-22.8)$ & & 0.887 \\
& $14.0(8.3-19.7)$ & & $0.90(0.54-1.19)$ \\
\hline
\end{tabular}


A

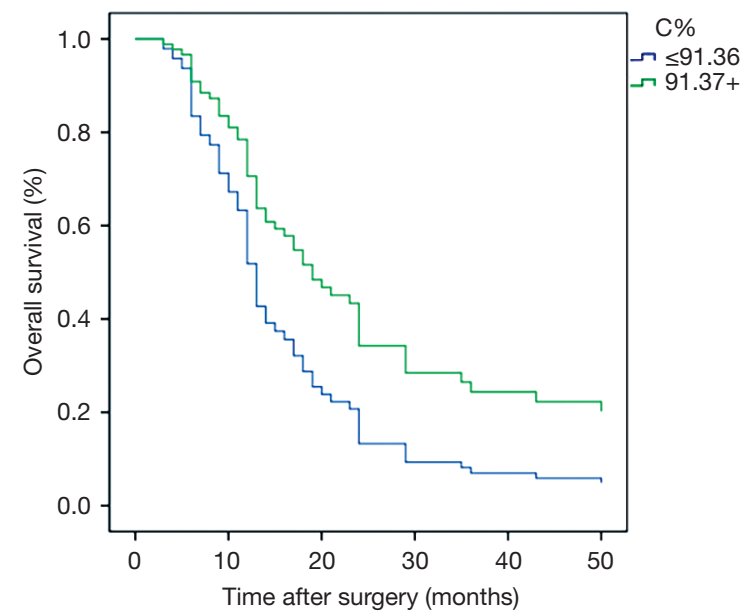

B

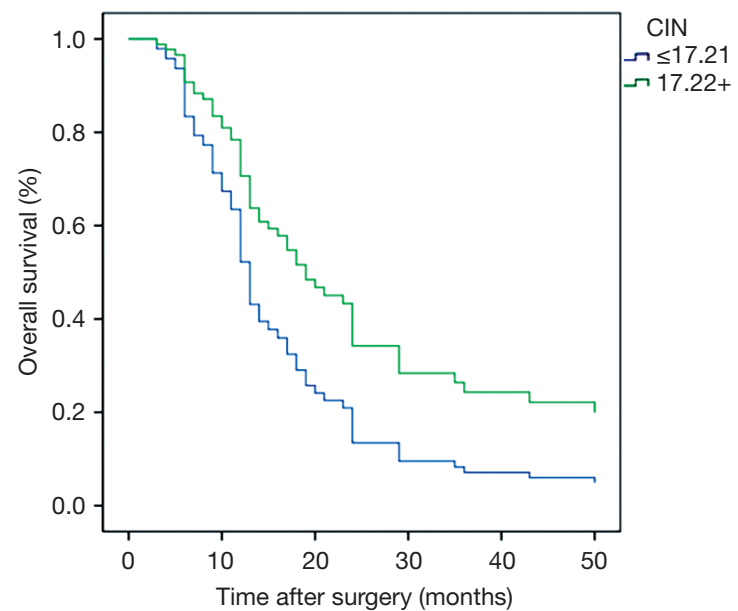

Figure 2 After multivariate analysis with survival, the results prompted that C\% (A) and Cin (B) were the independent prognostic factors. The death risk of cases with a high expression of CYP24A1 was lower than those with poor expressions.

Table 4 Primer sequence

\begin{tabular}{ll}
\hline Primer & Primer Sequence (5'-3') \\
\hline H-ACTIN-S & CACCCAGCACAATGAAGATCAAGAT \\
H-ACTIN-A & CCAGTTTTTAAATCCTGAGTCAAGC \\
H-CYP24A1-S & TACAGCGAACTGAACAAATGGT \\
H-CYP24A1-A & GAGTGACCATCATCCTCCCAA \\
\hline
\end{tabular}

features due to the limited sample size. This should be further researched. Secondly, only cell line was utilized for functional experiments. Thirdly, this study was only a single-center trial and the sample size was limited. Fourthly, there were not enough data about the proliferation, migration and invasion assays which should be further researched.

\section{Conclusions}

Through the experiment and the related literature, it is deemed that CYP24A1 has a considerable biological role in the occurrence, development, and prognosis of PDAC and the changes of its expression, to a certain extent, affect the differentiation and lymph node metastasis of PDAC, as well as the prognosis of PDAC patients. As an organ possessing both endocrine and exocrine functions, the pancreas has more various cell types in glands and PDAC contains a large number of stromal components, which make PDAC distinct from other solid tumors. The interaction between stromal cells and PDAC cancer cells has attracted significant attention. In all probability, CYP24A1 plays a role in PDAC in other ways than simply through the VD pathway. Conclusively, there is still a large number of questions about the role CYP24A1 in tumors (especially PDAC) that requires more research for its adequate interpretation.

\section{Acknowledgments}

Funding: None.

\section{Footnote}

Conflicts of Interest: All authors have completed the ICMJE uniform disclosure form (available at http://dx.doi. org/10.21037/tcr.2020.02.78). The authors have no conflicts of interest to declare.

Ethical Statement: The authors are accountable for all aspects of the work in ensuring that questions related to the accuracy or integrity of any part of the work are appropriately investigated and resolved. The study was conducted in accordance with the Declaration of Helsinki (as revised in 2013). The study was approved by the Ethical Committee of the General Hospital of PLA approved the present study (ID: S2016-098-02) and all subjects signed informed consent prior to the study. 
A

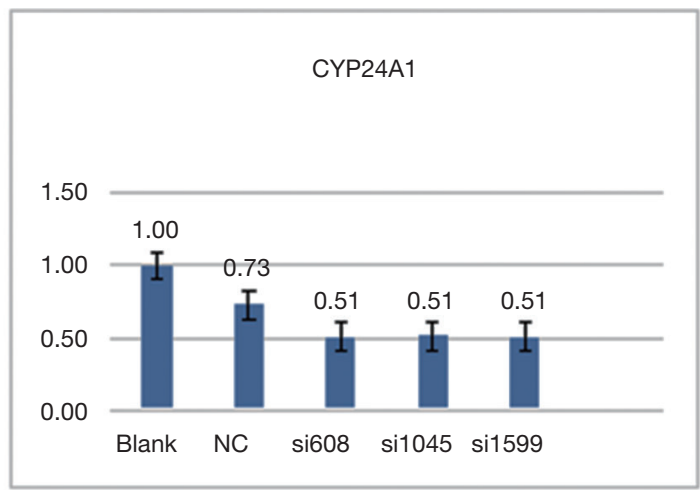

C

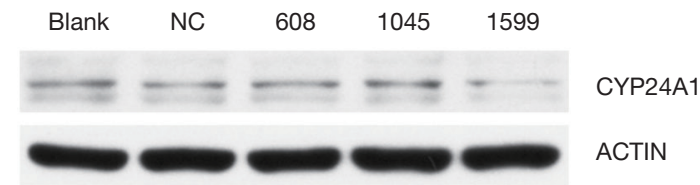

B

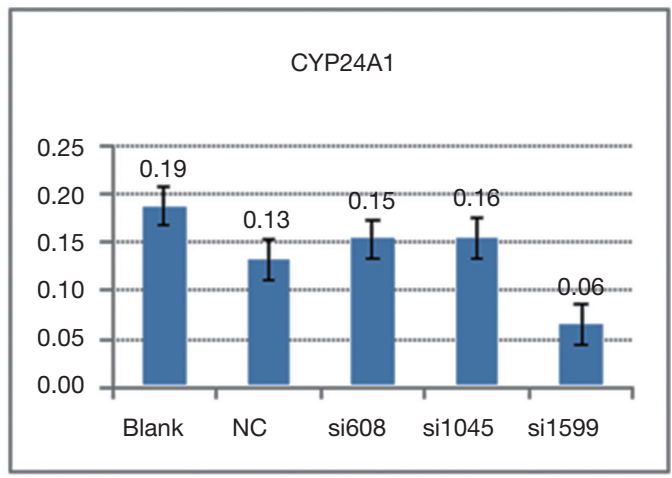

Figure 3 Silencing effects of CYP24A1 expression by transfection sequence (n=15). "Si606", "si1045" and "si1599" are three kinds of small interfering RNA sequences. (A) Change of mRNA levels; (B) change of protein levels; (C) protein bands.

Open Access Statement: This is an Open Access article distributed in accordance with the Creative Commons Attribution-NonCommercial-NoDerivs 4.0 International License (CC BY-NC-ND 4.0), which permits the noncommercial replication and distribution of the article with the strict proviso that no changes or edits are made and the original work is properly cited (including links to both the formal publication through the relevant DOI and the license). See: https://creativecommons.org/licenses/by-nc-nd/4.0/.

\section{References}

1. Chu LC, Goggins MG, Fishman EK. Diagnosis and Detection of Pancreatic Cancer. Cancer J 2017;23:333-42.

2. Zhao C, Gao F, Weng S, et al. Pancreaticcancer and associated exosomes. Cancer Biomark 2017;20:357-67.

3. Osanai M, Lee GH. CYP24A1-induced vitamin D insufficiency promotes breast cancer growth. Oncol Rep 2016;36:2755-62.

4. Yang J, Wang H, Ji A, et al. Vitamin D Signaling Pathways Confer the Susceptibility of Esophageal Squamous Cell Carcinoma in a Northern Chinese Population. Nutr Cancer 2017;69:593-600

5. Sun H, Wang C, Hao M, et al. CYP24A1 is a potential biomarker forthe progression and prognosis of human colorectal cancer. Hum Pathol 2016;50:101-8.

6. Tannour-Louet M, Lewis SK, Louet JF, et al. Increased expression of CYP24A1 correlates with advanced stages of prostate cancer and can cause resistance to vitamin D3based therapies. FASEB J 2014;28:364-72.

7. Rodriguez GC, Turbov J, Rosales R, et al. Progestins inhibit calcitriol-induced CYP24A1 and synergistically inhibit ovarian cancer cell viability: An opportunity for chemoprevention.Gynecol Oncol 2016;143:159-67.

8. Han SJ, Reis G, Kohanbash G, et al. Expression and prognostic impact of immune modulatorymolecule PD-L1 in meningioma. J Neurooncol 2016;130:543-52.

9. Kounnis V, Ioachim E, Svoboda M, et al. Expression of organic anion-transporting polypeptides 1B3, 1B1, and $1 \mathrm{~A} 2$ in humanpancreatic cancer reveals a new class of potential therapeutic targets. Onco Targets Ther 2011;4:27-32.

10. Hummel D, Aggarwal A, Borka K, et al. The vitamin D system is deregulated in pancreatic diseases. J Steroid Biochem Mol Biol 2014;144:402-9.

11. Jain A, Tiwari A, Verma A, et al. Vitamins for Cancer Prevention and Treatment: An Insight. Curr Mol Med 2017;17:321-40. 
12. Umar M, Sastry KS, Chouchane AI. Role of Vitamin D Beyond the Skeletal Function: A Review of the Molecular and Clinical Studies. Int J Mol Sci 2018;19:1618.

13. Jamali N, Sorenson CM, Sheibani N. Vitamin D and regulation of vascular cell function. Am J Physiol Heart Circ Physiol 2018;314:H753-65.

14. Li Z, Guo J, Xie K, et al. Vitamin D receptor signaling and pancreatic cancer cell EMT.Curr Pharm Des 2015;21:1262-7.

15. Mondul AM, Weinstein SJ, Layne TM, et al. Vitamin D and Cancer Risk and Mortality: State of the Science, Gaps, and Challenges. Epidemiol Rev 2017;39:28-48.

16. Arem H, Yu K, Xiong X, et al. Vitamin D metabolic pathway genes and pancreatic cancer risk. PLoS One 2015;10:e117574.

17. Zhang X, Huang XZ, Chen WJ, et al. Plasma 25-hydroxyvitamin D levels, vitamin D intake, and pancreatic cancer risk or mortality: a meta-analysis. Oncotarget 2017;8:64395-406.

Cite this article as: Gao W, Wang TF, Yi W, Tong WM. The effects of CYP24A1 on clinicopathological features and the prognosis of pancreatic ductal adenocarcinoma. Transl Cancer Res 2020;9(8):4476-4487. doi: 10.21037/tcr.2020.02.78
18. Wang K, Dong M, Sheng W, et al. Expression of vitamin $\mathrm{D}$ receptor as a potential prognostic factor and therapeutic target in pancreatic cancer. Histopathology 2015;67:386-97.

19. Ge N, Chu XM, Xuan YP, et al. Associations between abnormal vitamin $\mathrm{D}$ metabolism pathway function and non-small cell lung cancer. Oncol Lett 2017;14:7538-44.

20. Shan NL, Wahler J, Lee HJ, et al. Vitamin D compounds inhibit cancer stem-like cells and induce differentiation in triple negative breast cancer. J Steroid Biochem Mol Biol 2017;173:122-9.

21. Osanai M, Lee GH. CYP24A1-induced vitamin D insufficiency promotes breast cancer growth. Oncol Rep 2016;36:2755-62.

22. Höbaus J, Tennakoon S, Heffeter P, et al. Impact of CYP24A1 overexpression on growth of colorectal tumour xenografts in mice fed with vitamin D and soy. Int J Cancer 2016;138:440-50. 